\title{
ПОГРАНИЧНЫЕ ОПУХОЛИ ЯИЧНИКОВ У БЕРЕМЕННЫХ
}

\author{
А. А. Герасимова', М. В. Шамаракова', П. А. Клименко ${ }^{2}$ \\ ${ }^{1}$ Центр планирования семьи и репродукции, Москва, Россия \\ 2 Российский национальный исследовательский медицинский университет имени Н. И. Пирогова, Москва, Россия
}

\begin{abstract}
Пограничные опухоли яичников характерны для женщин репродуктивного периода, более чем у трети больных опухоли выявляют в возрасте 15-29 лет, средний возраст при первичной постановке диагноза состаляет 40 лет. Целью исследования было усовершенствовать методы диагностики пограничных опухолей яичников на фоне беременности и определить возможности выполнения органосохраняющего лечения. Обследовано 300 беременных с различными опухолевидными образованиями (ООЯ) и опухолями яичников (ОЯ), из которых 25 имели пограничные эпителиальные опухоли: 22 - серозные, три - муцинозные. До операции проводили УЗИ, определяли концентрацию в сыворотке крови CA-125, sFas, VEGF и IL6. Полученные результаты сопоставляли с морфологическими исследованиями. Проводили органосохраняющее и радикальное хирургическое лечение, при необходимости химиотерапию. При перекрестном сравнении изучали перинатальные исходы. Обнаружено, что различить доброкачественные опухоли яичников от пограничных (ПОЯ) и злокачественных (ЗОЯ) возможно с помощью УзИ и логрегрессионных моделей. Уровни VEGF выше 500 пг/мл, IL6 выше 8,1 пг/мл и СА-125 выше 300 ЕД/мл свидетельствуют о высокой вероятности ЗОЯ у беременных. И только морфологическое исследование тканей яичников, полученных независимо от хирургических способов, давало истинное представление о характере опухоли яичников у беременных. Вместе с тем у трех беременных с ОЯ при морфологическом исследовании выявлены участки ткани, характерные как для ПОЯ, так и для ЗОя. Таким образом, преобладание начальных форм опухолевого процесса, относительно благоприятное течение и прогноз при ПОЯ позволяют достаточно широко использовать хирургическое лечение щадящего характера с сохранением менструальной функции и фертильности.
\end{abstract}

Ключевые слова: ультразвуковое исследование, морфологическое исследование, опухоли яичников у беременных, CD31

Вклад авторов: все авторы внесли равнозначный вклад в проведение исследования и подготовку статьи, прочли и одобрили ее финальную версию перед публикацией.

Соблюдение этических стандартов: исследование одобрено этическим комитетом РНИМУ имени Н. И. Пирогова (протокол № 176 от 25 июня 2018 г.). Все пациенты подписали информированное согласие на участие в исследовании.

$\bigotimes$ Для корреспонденции: Петр Афанасьевич Клименко Севастопольский проспект, д. 24а, г. Москва, 117209; pa.klimenko@mail.ru

Статья получена: 07.04.2020 Статья принята к печати: 21.04.2020 Опубликована онлайн: 26.04.2020

DOI: $10.24075 /$ vrgmu.2020.023

\section{BORDERLINE OVARIAN TUMORS IN PREGNANCY}

\author{
Gerasimova $\mathrm{AA}^{1}$, Shamarakova MV1ำ Klimenko PA²凶
}

${ }^{1}$ Center of Family Planning of Moscow Department of Health, Moscow, Russia

${ }^{2}$ Pirogov Russian National Research Medical University, Moscow, Russia

\begin{abstract}
Borderline ovarian tumors (BOTs) are common in women in their reproductive years. In more than one-third of patients tumors are detected at the age of 15-29, the average age at initial diagnosis is 40 . The study was aimed to improve methods for BOTs diagnosis in pregnancy and to determine the possibilities of organ preservation treatment. A group of 300 pregnant women with various tumor-like formations and ovarian tumors was examined. Of them, 25 patients had borderline epithelial tumors (22 patients had serous and 3 patients had mucinous tumors). Ultrasound examination together with blood serum CA-125, sFas, VEGF and IL6 level assessment were performed prior to surgery. The results obtained were compared with the results of morphological studies. Organ preservation and radical surgical treatment were carried out, and chemotherapy, if necessary. Perinatal outcomes were studied when performing the cross-comparison. It was discovered, that ultrasonography and logistic regression analysis made it possible to distinguish between benign ovarian tumors, BOTs and malignant ovarian tumors. The levels of VEGF above the $500 \mathrm{pg} / \mathrm{ml}$, IL6 above the $8.1 \mathrm{pg} / \mathrm{ml}$ and CA-125 above the $300 \mathrm{U} / \mathrm{ml}$ indicated the high probability of malignant ovarian tumors in pregnant women. Only the morphological study of ovarian tissue, obtained regardless of surgical methods, ensured understanding of the ovarian tumor's true nature during pregnancy. At the same time, in three pregnant women with ovarian tumors, the morphological examination revealed some tissue areas common both for BOTs and malignant ovarian tumors. Thus, the predominance of the tumor early stages, relatively mild course and, favorable prognosis in patients with BOTs make it possible to use gentle surgical treatment making it possible to preserve menstrual function and fertility.
\end{abstract}

Keywords: ultrasound, morphological examination, ovarian tumors in pregnant women, CD31

Author contribution: all authors contributed to the research and manuscript preparation equally, read the approved the final version of the article before publishing.

Compliance with ethical standards: the study was approved by the Ethics Committee of Pirogov Russian National Research Medical University (protocol № 176 dates June 25, 2018). The informed consent was submitted by all study participants.

$\square$ Correspondence should be addressed: Piotr A. Klimenko

Sevastopolsky prospect, 24a, Moscow, 117209; pa.klimenko@mail.ru

Received: 07.04.2020 Accepted: 21.04.2020 Published online: 26.04.2020

DOI: 10.24075/brsmu.2020.023

Пограничные эпителиальные опухоли (ПОЯ) представляют собой овариальные новообразования с наличием клеточной и ядерной атипии без деструктивной стромальной инвазии и имеют благоприятный прогноз. Среди всех неоплазий яичников ПОЯ составляют 15-20\% [1-3]. Однако при анализе данных специализированных онкологических клиник выявлена большая частота $-21-35 \%$ в связи с профильным отбором больных [4-8]. У беременных частота злокачественных образований яичников (ЗОЯ) вместе с ПОЯ не превышает 9\%. Из-за отсутствия патогномоничных симптомов, достоверных признаков эхографии и результатов определения маркерного гликопротеина СА125 клиническая диагностика ПОЯ затруднена, и зачастую возникают сложности с дифференцированием их от 
доброкачественных и злокачественных видов неоплазий яичников. Поэтому диагноз пограничной опухоли может быть достоверно установлен только по итогам послеоперационного морфологического исследования [9-10]. Более чем у 70\% беременных опухоли выявляют при проведении ультразвукового исследования (УЗИ) на ранних сроках гестации и они соответствуют начальным стадиям по классификации FIGO. Хирургическое лечение ЗОЯ и ПОЯ у беременных выполняют, как правило, в первом и втором триместрах беременности [5, 11-12], что ведет к росту перинатальной заболеваемости и ранней детской смертности.

Целью исследования было усовершенствовать методы диагностики ПОЯ на фоне беременности и определить возможности выполнения органосохраняющего лечения.

\section{ПАЦИЕНТЫ И МЕТОДЫ}

За период с 2000 по 2017 г. были проспективно обследованы 300 беременных с различными опухолевидными образованиями (ООЯ) и опухолями яичников (ОЯ). Критерии включения пациенток в исследование: беременные с ООЯ/ОЯ в I-ІІІ триместрах. Критерии исключения: отказ беременной от участия в исследовании; беременные с установленным до начала исследования онкологическим заболеванием; пациентки с угрозой прерывания беременности, внутриутробным инфицированием, пренатальными повреждениями плода, установленными до проведения исследования. Результать исследования оценивали при перекрестном анализе. Распределение их в зависимости от морфологической структуры, стадии опухолевого процесса и степени дифференцировки представлено на рис. 1.

У 76 из 300 беременных с новообразованиями яичников были выявлены пограничные (ПОЯ) и злокачественные (ЗОЯ) опухоли. Среди 25 ПОЯ серозная форма зарегистрирована в 22 наблюдениях, муцинозная в трех. Следует отметить, что исследование проводили длительное время и набор пациентов носил случайный, не популяционный характер.

УзИ проводили на аппарате Voluson 530 МТ (Kretztechnik; Австрия) и Voluson E8 (General Electric;
США) с использованием датчиков: RIC5-9-D (4-9 МГц), C1-5-D (2-5 МГц), RAB4-8-D (2-8 МГц). Комплексное УЗИ выполняли в режиме 2D и 3D в комбинации с использованием допплерографических методик в режиме цветового и энергетического картирования (ЦДК и ЭДК), а также трехмерной ангиограсии. При ЦДК исследовали ряд параметров: характер сосудистого рисунка (по периферии, в центральной части опухоли, в перегородках, в папиллярных разрастаниях), анализ кривой скорости кровотока (KCK) с определением показателя сосудистого сопротивления индекса резистентности (resistance index, Rl) и максимальной систолической скорости кровотока (МСС, см/с). Из 30 сонографических признаков ООЯ, доброкачественных образований (ДОЯ), ПОЯ и ЗОЯ информативными оказались 17. Для УЗ-диагностики использовали предложенную нами модель, разделяющую ДОЯ от ПОЯ и ЗОЯ [13]. В предыдущих наших исследованиях [14] было показано, что опухоли яичников у беременных имеют УЗ-признаки, с помощью которых их можно с довольно высокой точностью дифференцировать на ДОЯ и ЗОЯ. В ходе исследования было обнаружено, что УЗ-особенности различных ОЯ имеют статистически значимые различия. При изучении эхографических признаков злокачественных эпителиальных опухолей яичников (рака яичников, или РЯ) выделено четыре типа строения, и что самое важное - своеобразие гемодинамических показателей. Одновременно была создана экспертная шкала на основании анализа УЗ-показателей. Для оценки точности модели помимо собственно процента верных отнесений учитывали параметры чувствительности (Se) и специсричности (Sp).

Молекулярно-биологические исследования проводили по следующей схеме. Концентрацию СА-125 определяли с помощью иммуноферментного анализа с использованием тест-системы (Siemens; Германия). В сыворотке крови концентрацию sFas измеряли иммуноферментным методом с использованием моноклональных антител, а концентрацию VEGF — иммуноферментным методом при использовании наборов реактивов («R@D»; CШA). Определение концентрации IL6 производили иммуноферментным методом ELISA «сэндвичевого» типа с использованием наборов реактивов («R\&D»; CШA).

гистологическая структура новообразований яичников

\begin{tabular}{cccc}
$46 \%$ & $31 \%$ & $17 \%$ & $7 \%$ \\
\hline Доброкачественные опухоли & — Опухолевидные образования & \\
Злокачественные опухоли & Пограничные опухоли & \\
Гистологическая структура злокачественных опухолей яичников & $30 \%$ & $8 \%$ \\
\hline $62 \%$ & 30
\end{tabular}

- Рак яичников - Пограничные опухоли Неэпителиальные зоя

Степень дифференцировки

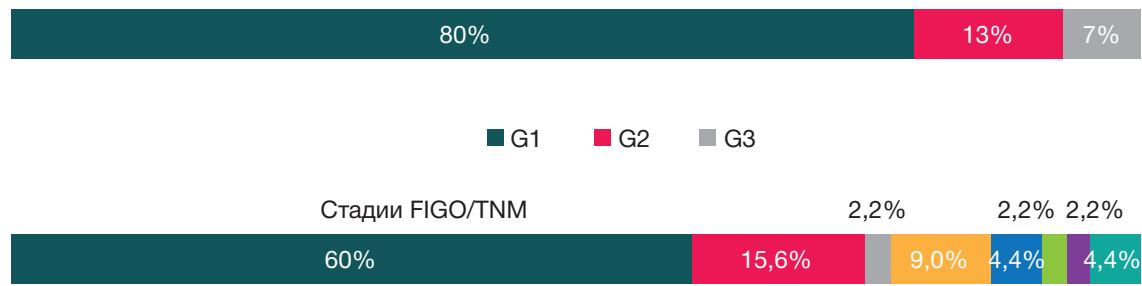

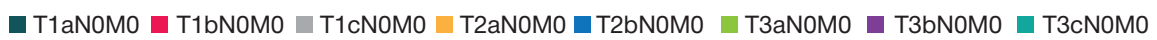

Рис. 1. Распределение ОЯ/ООЯ в зависимости от гистологической структуры, стадий опухолевого процесса (ПОЯ/ЗОЯ) и степени диффференцировки (РЯ) 
Гистологические препараты, окрашенные гематоксилинэозином, оценивали разные патологи. При постановке морфологического диагноза использовали классификацию опухолей женской репродуктивной системы ВОЗ 2003 г., поскольку именно она была принята на территории РФ во время проведения исследования. Для иммуногистохимического исследования были отобраны парафиновые блоки у 15 беременных с ПОЯ и у 10 - с ЗОЯ. Ангиогенез анализировали с помощью антител к фактору роста эндотелия сосудов (vascular endothelial growth factor, VEGF) - главному сигнальному белку ангиогенеза (VENTANA; CША) и эндотелиальному маркеру CD31 - молекуле межклеточной адгезии тромбоцитов и эндотелия типа 1 (клон JC70; VENTANA, США). При оценке экспрессии CD31 сначала при малом увеличении микроскопа были отобраны участки с наибольшим числом микрососудов. В дальнейшем в двух отдельных полях зрения с повышенной микрососудистой площадью при 200-кратном увеличении микроскопа подсчитывали число всех позитивных микрососудов. Уровень экспрессии VEGF оценивали полуколичественным методом в пяти полях зрения при 400-кратном увеличении микроскопа, включающим сопоставление интенсивности окрашивания и числа позитивных клеток. При измерении интенсивности окрашивания неокрашенные клетки соответствовали 0 баллам, клетки со слабожелтым окрашиванием - 1 баллу, клетки с желто-коричневым окрашиванием - 2 баллам, клетки с коричневым окрашиванием - 3 баллам. Число позитивно окрашенных клеток варьировало: 0 баллов менее 10\% всех клеток, 1 балл - 10-49\% окрашенных клеток, 2 балла - 50-74\% окрашенных клеток, 3 балла более $75 \%$ окрашенных клеток. Результаты обоих подсчетов складывали, значение более 2 баллов считали положительным.

Помимо этого, были изучены истории болезни и исходы беременности и родов у 300 пациенток с новообразованиями яичников после проведенного лечения.
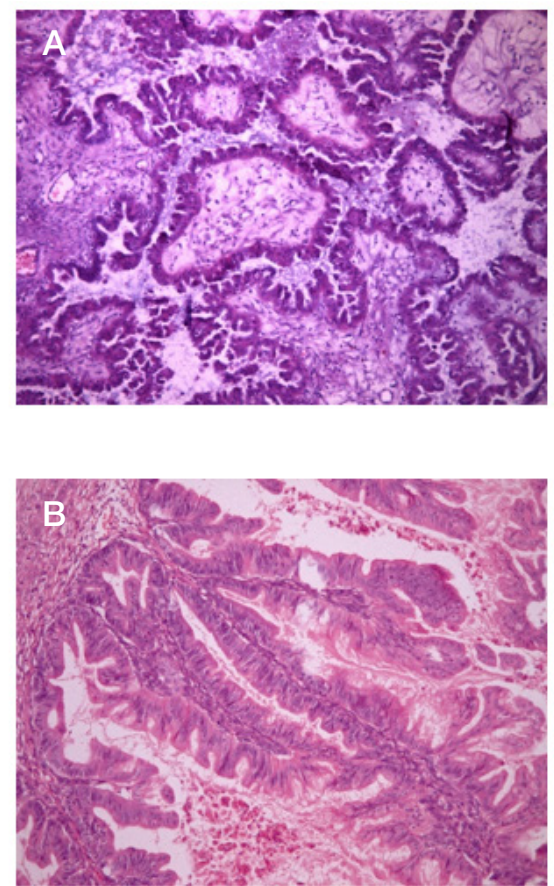

Для статистической обработки данных применяли прикладной пакет программ SPSS 15.0 (IBM; CША). Данные подвергали частотному анализу путем построения кросс-таблиц. Различия считали статистически значимыми при значении $p<0,05$.

\section{РЕЗУЛЬТАТЫ ИССЛЕДОВАНИЯ}

Проведенные исследования показали, что клинические характеристики обследованных беременных существенно не различались по группам. Так, возраст у 76 беременных с ПОЯ и ЗОЯ варьировал в широком диапазоне, от 18 до 45 лет. Более чем в 60\% наблюдений он составлял 30 лет. У беременных с ПОЯ/3ОЯ были отмечены боли внизу живота и нарушение функций соседних органов в 9\% случаев, увеличение объема живота - в 10,9\%, в анамнезе были выявлены нарушение менструального цикла в 10,9\% и бесплодие - в 2,7\%. Структура сопутствующих экстрагенитальной, гинекологической патологий и перенесенные гинекологические операции до настоящей беременности у пациенток с ООЯ/ОЯ в большей степени коррелировали с возрастом и не зависели от морфологической структуры опухолей.

Среди гистологических форм ПОЯ преобладали серозные - у 22 (88\%) пациенток, муцинозные типы встречались у 3(12\%) беременных. Билатеральное поражение яичников было установлено в 28\% наблюдений. Большинство ПОЯ на фоне беременности диагностированы в I стадии опухолевого процесса - у 19 (76\%) пациенток, II стадия выявлена у 5 (20\%) больных и только в одном наблюдении верифицирована III стадия.

УЗ-признаки у беременных с пограничными опухолями яичников соответствовали нескольким вариантам строения: в 32,6\% наблюдений констатирован смешанный тип строения опухоли с преобладанием солидного компонента, примерно у 55\% больных отмечалось преобладание кистозного компонента, к солидным опухолям отнесены более 10\%. При допплерографии
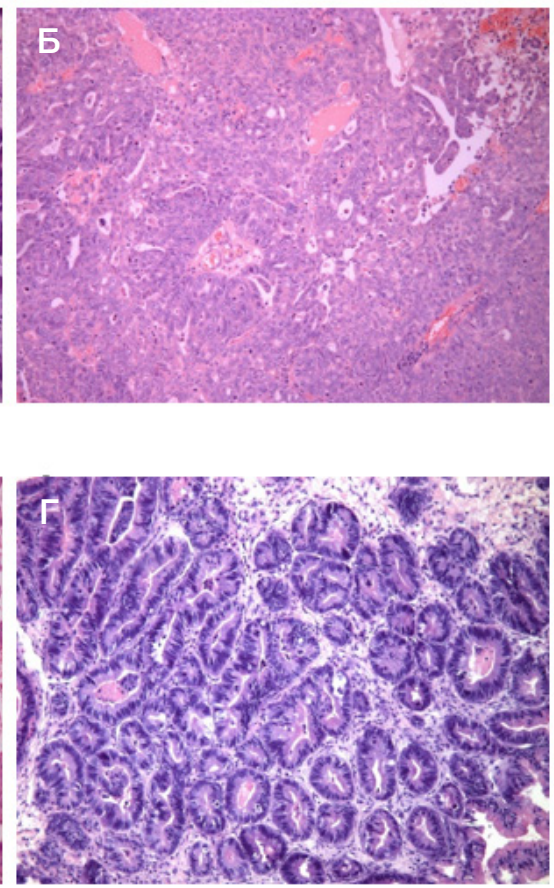

Рис. 2. Морфологическая картина ПОЯ и зОЯ яичников у беременных. А. Серозная пограничная цистаденома яичника (×10, окраска гематоксилинэозином). Б. Серозная низкодифференцированная карцинома яичника (×10, окраска гематоксилин-эозином). В. Муцинозная пограничная цистаденома яичника (×10, окраска гематоксилин-эозином). Г. Муцинозная карцинома яичника (×10, окраска гематоксилин-эозином) 
была выявлена центральная и периферическая гиперваскуляризация с низкими значениями Rl (меньше или равно 0,4) и высокими показателями MCC (более 15 cм/c) при оценке KCK, с наличием мозаичного типа кровотока, свидетельствующего о присутствии артериовенозного шунтирования в сосудистой сети опухоли.

Использование предложенной нами модели для дифференциальной диагностики ОЯ у беременных позволило отличать ООЯ и ДОЯ от ПОЯ и ЗОЯ (чувствительность составила 100\%, специфичность 92,3\% при суммарной точности модели 92,8\%). Из-за выраженной схожести изображения и гемодинамических показателей при проведении комплексной эхографии не удалось дифференцировать ПОЯ и ЗОЯ. Вместе с тем при этих неоплазиях во всех наблюдениях были выявлены центральное расположение сосудов с разветвленной сетью в перегородках, солидном компоненте, папиллярных разрастаниях и низкорезистентный кровоток.

При ПОЯ концентрация СА-125 в крови беременных варьировала от 24,4 до 361 ЕД/мл в I триместре и от 24,1 до 223 ЕД/мл - во ॥ триместре беременности. Уровень sFas составлял 40-200 нг/мл в I триместре и 46-180 нг/мл во II триместре беременности. Концентрация VEGF колебалась от 89 до 286 пг/мл в I триместре и от 92 до 480 пг/мл во II триместре беременности. IL6 достигали 3,6-12 пг/мл В I триместре и 8-40,9 пг/мл во II триместре беременности.

При ЗОя было отмечено достоверное повышение по сравнению с ПОЯ в сыворотке крови как СА-125, так и других маркеров канцерогенеза - sFas, VEGF, IL6 в любые сроки беременности. В крови трех пациенток с аденокарциномой яичников СА-125 составили 540-1224,6 ЕД/мл, sFas - 180-312,6 нг/мл, VEGF - 510-1028 пг/мл, IL6 9,8-40,9 пг/Мл. Аналогичную концентрацию молекулярнобиологических факторов наблюдали в крови пациенток с дисгерминомой, смешанной герминогенной опухолью и незрелой тератомой. В этих наблюдениях уровень СА-125 превышал 361 ЕД/мл, sFas — 240 нг/Мл, VEGF — 490 пг/мл, IL6 - 8,1 пг/мл.

При морфологическом исследовании ПОЯ (рис. 2) в 22 наблюдениях были зарегистрированы признаки, позволяющие дифференцировать их как от ДоЯ, так и от 3ОЯ. В трех наблюдениях были обнаружены несовпадения в интерпретации окончательного гистологического ответа у больных, которым был установлен диагноз серозной аденокарциномы на фоне серозной пограничной опухоли. При повторном пересмотре препаратов элементов злокачественной опухоли не было обнаружено.

Пограничная серозная цистаденома представляла собой кистозную опухоль с рыхлой стенкой и выраженными папиллярными разрастаниями, которые занимали всю внутреннюю поверхность и в 70\% случаев определялись и на наружной поверхности. ПОЯ отличались эпителиальными разрастаниями с образованием клеточных пучков и отпочкованием групп клеток одновременно со строго упорядоченным ветвлением, при котором мелкие папиллы происходят от крупных, центрально расположенных сосочков. Клетки пограничных серозных опухолей имели особенности эпителиальной и мезотелиальной дифференцировки. Реснитчатые клетки были похожи на клетки маточной трубы и были выявлены в трети опухолей. Клетки с обильной эозинофильной цитоплазмой и округлыми ядрами имели сходство с мезотелием и располагались на верхушках папилл. Ядра клеток располагались базально, овальной или округлой формы, с легкой атипией, нежным хроматином и иногда выраженными ядрышками. Выявлены редкие митозы (обычно 4 в 10 полях зрения). Псаммомные тельца обнаружены в половине наблюдений.

Серозные карциномы достигали больших размеров (до 20 см в диаметре), представляли собой кисты с серозным или сукровичным содержимым, заполненные мягкими рыхлыми папиллярными разрастаниями. Наружная поверхность была гладкая, изредка с папиллярными структурами. Опухоли солидного строения обычно имели менее выраженные папиллы, розово-серого цвета, были мягкими или плотными в зависимости от характера подлежащей стромы. Одновременно наблюдались кровоизлияния и некрозы. При микроскопическом исследовании серозные карциномы имели папиллярное с очагами солидного строение, увеличенные округлые клетки с полиморфными, гиперхромными ядрами, глыбчатым ядерным хроматином и увеличенным ядерно-цитоплазматическим соотношением, псевдомногорядность эпителия, характеризовались потерей полярности, отсутствием ресничек на поверхности клеток, повышенной митотической активностью.

Пограничная муцинозная цистаденома яичника была, как правило, многокамерная, диаметром до 30 см, содержала соломенного цвета жидкость или слизь. Морфологическое изучение препаратов этих опухолей выявляло участки, выстланные многорядным муцинозным эпителием кишечного типа с образованием ворсинчато-железистых и папиллярных структур с легкой степенью атипии ядер клеток.

Муцинозная карцинома отличалась от пограничной муцинозной цистаденомы наличием очагов с комплексным расположением желез, выстланных клетками с умеренной и тяжелой атипией ядер, митозами, а также присутствием очагов некроза в опухоли.

Экспрессия CD31 (рис. 3-4) была выявлена в строме опухолей во всех случаях. Среднее число CD31 позитивных сосудов у женщин с ПОЯ составило 36 (от 12 до 48), у пациенток с ЗОЯ - 44 (от 19 до 56). Иммунореактивность в отношении маркера VEGF, оцененная полуколичественным методом, у женщин с ПОЯ соответствовала 5 баллам (от 4 до 6), у пациенток с ЗОЯ - 6 баллам (от 5 до 7). Достоверные различия при исследовании экспрессии обоих маркеров не были установлены.

Анализ историй болезни беременных с ПОЯ и ЗОЯ показал, что части из них при распространенном опухолевом процессе проведены полные циторедуктивные операции с прерыванием беременности. Другим пациенткам циторедуктивные операции проводили дважды: при обнаружении опухоли и после операции кесарева сечения.

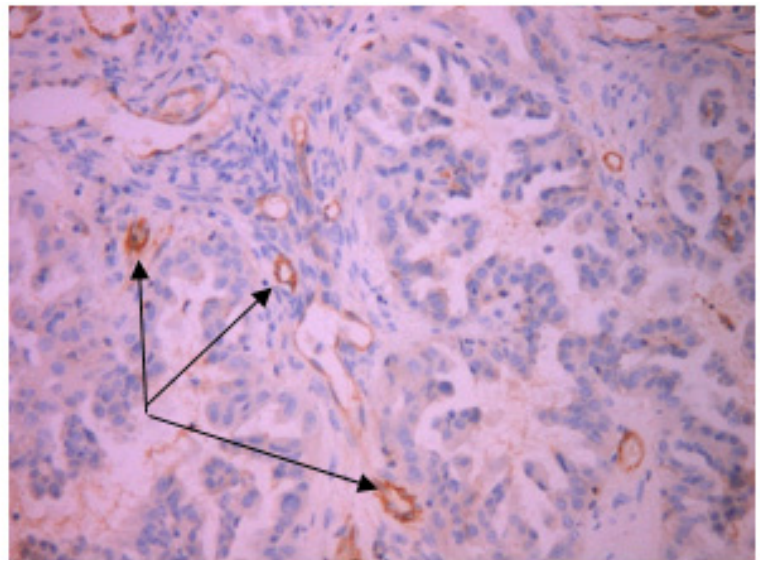

Рис. 3. Экспрессия CD31 в ЗОЯ (×20). Стрелками указаны сосуды 
У всех пациенток с признаками озлокачествления опухолей яичников проводили срединную лапаротомию с обходом пупка слева. В шести наблюдениях была выполнена сначала диагностическая лапароскопия, а затем в связи с подозрением на РЯ - лапаротомия и удаление первичного очага.

Объем хирургического вмешательства определяли интраоперационно в соответствии с данными клинической картины заболевания, репродуктивного анамнеза и возрастом пациентки, результатами УЗИ, уровнями онкомаркеров в сыворотке крови и результатами срочного гистологического исследования. В ходе операций проводили хирургическое стадирование опухолевого процесса, ревизию органов брюшной полости и малого таза, резекцию/удаление большого сальника, множественные биопсии брюшины, взятие смывов или асцитической жидкости из брюшной полости. При муцинозном типе опухоли проводили аппендэктомию. У больных, не заинтересованных в сохранении беременности и фертильности, выполняли радикальные операции в семи из 76 наблюдений. На первом этапе во время беременности 20 пациенткам с ПОя было выполнено органосохраняющее оперативное вмешательство с сохранением матки и части здорового яичника. В двух случаях проводили двухстороннюю аднексэктомию. В одном из них пограничная опухоль стала находкой после гистологического исследования резецированной части визуально неизмененного контрлатерального яичника (IB стадия).

Следует отметить, что при гистологическом исследовании биопсийного материала или препаратов опухоли могут возникать ошибки и неточности. Так, в наших наблюдениях у трех больных с ОЯ на фоне беременности при морфологическом исследовании зарегистрированы участки ткани, характерные как для ПОЯ, так и для ЗОЯ. Был установлен диагноз высокодифференцированной аденокарциномы обоих яичников на фоне серозной цистаденомы пограничного типа. У одной из них в сроки 11-12 недель беременности клинически определены двухсторонние опухоли яичников с признаками озлокачествления, асцит. В онкологическом стационаре после проведения диагностической лапароскопии, правосторонней аднексэктомии с экспрессгистологическим исследованием было сделано заключение: пограничная цистаденома. Выполнена конверсия лапароскопического доступа на лапаротомический. Путем срединной лапаротомии были выполнены биопсия левого яичника, резекция большого сальника, множественные биопсии брюшины. Морфологически установлен диагноз высокодифференцированной аденокарциномы, развившейся на фоне серозной пограничной опухоли с раковыми эмболами в просвете сосудов большого сальника (рак яичников T3сNOMO). Выполнены искусственное прерывание беременности и радикальная операция: экстирпация матки с левыми придатками, субтотальная резекция большого сальника. При цитологическом исследовании смывов из брюшной полости выявлены элементы аденогенного рака. Перед назначением химиотерапии был проведен междисциплинарный онкологический консилиум в связи с расхождением в трактовке результатов цитологического и гистологического исследований разными специалистами. Первичный диагноз не был подтвержден. Установленный диагноз: пограничная опухоль яичников с неинвазивными имплантами в большом сальнике. От проведения химиотерапии было решено отказаться. Больная наблюдается четыре года без признаков прогрессирования заболевания.

Результаты лечения пациенток с пограничными опухолями были следующие: у трех беременных проведено прерывание беременности с проведением хирургического лечения в объеме пангистерэктомии из-за наличия аденокарциномы на фоне серозной ПОЯ, у двух беременных произошли выкидыши, 10 пациенток родили самостоятельно в срок, шесть беременных родоразрешены раньше срока путем операции кесарева сечения при появлении акушерских показаний, в четырех случаях повторные операции проведены с целью рестадирования.

У беременных с ПОЯ впоследствии возникли рецидивы опухоли в двух случаях. У одной при серозном гистотипе опухоли ІА стадии в ткани резецированного яичника после органосохраняющей операции рецидив выявлен на пятом году наблюдения. При морфологическом исследовании была обнаружена высокодифференцированная аденокарцинома, после чего проведена радикальная операция, дополненная химиотерапией. Во втором наблюдении через 2 года после первой операции возник рецидив, идентичный по гистологической структуре первичной опухоли (атипически пролиферирующая опухоль серозного типа). После удаления рецидивного новообразования было проведено комбинированное лечение. Обе пациентки живы более 3 лет. Выбыли из-под наблюдения 5 пациенток. Отдаленные результаты лечения нами прослежены у 17 из 25 больных в течение 3-10 лет. Все наблюдаемые живы на момент проведения исследования. Общая пятилетняя выживаемость составила 100\%.

У пациенток с ПОЯ через 2-5 лет после проведенного оперативного вмешательства наступило 9 беременностей, 4 из которых закончились родами с благоприятным исходом. В 3 наблюдениях беременности закончились самопроизвольным абортом.

\section{ОБСУЖДЕНИЕ РЕЗУЛЬТАТОВ}

Данные литературы свидетельствуют об отсутствии специфических клинических проявлений ПОЯ при беременности. Комплексная сонография с использованием допплерометрических методик, включенных в модели для дифференциальной диагностики, имеет высокую специфичность.

В настоящее время не выделено молекулярнобиологических факторов, достоверно характеризующих ПОЯ [2, 15]. Применение большинства онкомаркеров ограничено из-за высокой вариабельности их показателей,

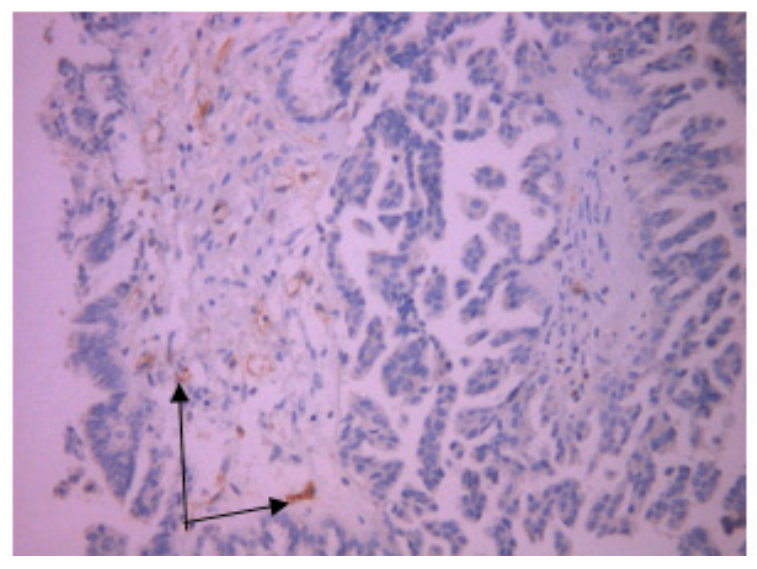

Рис. 4. Экспрессия CD31 в ПОЯ (×20). Стрелками указаны сосуды 
в том числе и в зависимости от срока гестации. В проведенном исследовании значительное повышение уровней маркеров канцерогенеза выше пороговых (VEGF — выше 500 пг/мл, IL6 - 8,1 пг/мл) выявлено при наличии злокачественных неоплазий яичников у беременных. При этом специфичность теста составила 91,5\%, чувствительность - 75\%. Концентрация СА125 при ЗОЯ у беременных превышала 300 ЕД/мл. Эти результаты согласуются с данными других авторов [16].

При оценке уровня экспрессии VEGF в парафиновых блоках полуколичественным методом повышенная иммунореактивность в отношении данного маркера, соответствующая 5-7 баллам, была зарегистрирована в карциномах яичников. Ассоциация экспрессии VEGF и овариального рака подтверждена многими работами. Доказано повышение иммунореактивности VEGF в карциноме яичника по сравнению с ПОЯ, при этом высокий уровень экспрессии VEGF свидетельствует о прогрессировании заболевания [17]. Повышенная иммунореактивность CD31 в препаратах ЗОЯ по сравнению с образцами ПОЯ указывает на усиленный кровоток в опухолевой ткани, обусловленный неоваскуляризацией, регистрируемой в злокачественных опухолях [18].

Основным методом лечения ПОЯ с органосохраняющим или радикальным подходом остается хирургический метод. В мировой литературе активно обсуждают вопрос о возможности ультраконсервативных вмешательств как органосохраняющего варианта с сохранением неизмененной ткани яичника, пораженного ПОЯ, путем выполнения резекции/цистэктомии [2, 19]. Оптимальным объемом операции считается аднексэктомия на стороне поражения с морфологическим исследованием смывов с брюшины и ее множественных биоптатов; окончательное хирургическое стадирование следует проводить во время кесарева сечения или после родов, если родоразрешение проводили через естественные родовые пути [20, 21]. В нашем исследовании ультраконсервативные вмешательства не были выполнены, в 80\% наблюдений у пациенток с ПОЯ применяли органосохраняющее хирургическое лечение. С целью рестадирования повторные операции были выполнены у 16\% наблюдаемых.

Примерно у 1/3 больных необходимо проведение окончательного послеоперационного морфологического исследования на парафиновых блоках при пОя и высокодифференцированной аденокарциноме [2, 22-24]. По некоторым данным, высокая частота гипердиагностики при наличии фокусов, подозрительных на рак яичников при ПОЯ, даже при проведении окончательного гистологического исследования в специализированных учреждениях, приводит к необоснованному завышению объемов хирургических вмешательств [3]. По нашим результатам, несовпадения в трактовке морфологического ответа при дифференциальной диагностике ПОЯ и РЯ обнаружены в 12\% случаев. Разнообразное строение ПОЯ, необходимость тщательного исследования множественных срезов обусловливают повышенные требования к квалификации и опыту морфолога, аналогичного мнения придерживаются и другие исследователи [3, 9, 22].

Общая частота рецидивов при ПОЯ варьирует от 3 до 10\%, при распространенных стадиях рецидивы развиваются у 25\% больных. В проведенном нами исследовании рецидивы были выявлены в 8\% наблюдений. По результатам публикаций, пятилетняя выживаемость при I-II стадиях составляет около 98-99\%, при III-IV стадиях 82-90\% [25, 26]. Возможно, столь высокие показатели пятилетней выживаемости в выполненном исследовании связаны с выявлением ПОЯ в начальных стадиях процесса и малым количеством исследуемых групп.

В работах по изучению фертильности после органосохраняющего лечения сообщается, что спонтанные беременности возникают в 40-72\% наблюдений. Влияние беременности на течение заболевания не установлено [1, 2, 27, 28]. Следует отметить, что репродуктивными результатами, полученными в нашей работе, были зафиксированные беременности более чем у 35\% пациенток после органосохраняющих оперативных вмешательств при выявлении ПОЯ на фоне беременности.

Полученные нами результаты позволили выделить в диагностическом алгоритме обследования беременных с подозрением на малигнизированные ОЯ совокупность таких признаков, как смешанное эхографическое строение с гиперваскулярным типом кровотока и низкими показателями индексов сосудистого сопротивления, значения VEGF, превышающие 500 пг/мл, и IL6 более 8,1 пг/мл, концентрация СА-125 выше 300 ЕД/мл, как наиболее важные. Однако схожесть УЗ-признаков ПОЯ и ЗОЯ не позволила однозначно дифференцировать данные типы неоплазий. Диагноз ПОЯ является прерогативой окончательного послеоперационного морфологического исследования. Результаты срочного гистологического анализа тканей яичников на замороженных срезах не во всех наблюдениях позволяют получить истинное представление о характере ОЯ у беременных. Высокие показатели общей пятилетней выживаемости после выполнения хирургического лечения ПОЯ в органосохраняющем объеме на фоне беременности в проведенном исследовании демонстрируют возможность реализации таких щадящих подходов при начальных стадиях.

\section{ВЫВОДЫ}

Несмотря на значительный научный и практический интерес к ПОЯ многие проблемы, касающиеся совершенствования диагностики и методов лечения больных во время беременности, далеки от своего решения. Преобладание начальных форм опухолевого процесса, относительно благоприятное течение и прогноз при ПОя позволяют достаточно широко использовать щадящий характер хирургического лечения с сохранением менструальной функции и фертильности.

\section{Литература}

1. Батталова Г. Ю. Пограничные опухоли яичников (оптимизация методов лечения и медико-социальной реабилитации больных) [диссертация]. М., 2005.

2. Новикова Е. Г., Андреева Ю. Ю., Шевчук А. С. Пограничные опухоли яичников. Онкология. 2013; (1): 84-91.
3. Давыдова И. Ю. Серозные пограничные опухоли яичников (клинико-морфологические особенности, лечение, прогноз) [диссертация]. М., 2018.

4. Giuntoli RL, Vang RS, Bristow RE. Evaluation and management of adnexal masses during pregnancy. Clin Obstet Gynecol. 2006; 49 
(3): 492-505.

5. Бахидзе Е. В. Опухоли яичника у беременных. Журнал акушерства и женских болезней. 2011; 3: 190-6.

6. Morice P, Uzan C, Gouy S, Verschraegen C, Haie-Meder C Gynecological cancers in pregnancy. Lancet. 2012; 379 (9815); 558-69.

7. Aggarwal $\mathrm{P}$, Kehoe $\mathrm{S}$. Ovarian tumors in pregnancy a literature review. Eur J Obstet Gynecol Reprod Biol. 2011; 155 (2): 119-24.

8. Gui T, Cao D, Shen K, Yang J, Fu C, Lang J, Liu X. Management and outcome of ovarian malignancy complicating pregnancy: an analysis of 41 cases and review of the literature. Clin Transl Oncol. 2013; 15 (7): 548-54.

9. Шлома Е. Н., Фридман М. В., Шелкович С. Е., Демидчик Ю. Е. Пограничные эпителиальные опухоли яичников: клиническое течение и проблемы морфологической диагностики. Минск: Изд-во «Бел МАПО», 2012; 80 с.

10. Давыдова И. Ю., Кузнецов В. В., Карселадзе А. И., Мещерякова Л. А. Пограничные опухоли яичников. Акушерство и гинекология: новости, мнения, обучение. 2019; 7 (1): 92-104.

11. Nadereh B, Mojgan KZ, Mitra MG, Fatemeh G, Azamsadat M, Fahimeh G. Ovarian carcinoma with pregnancy: a clinicopathologic analysis of 23 cases and review of the literature. BMC Pregnancy Childbirth. 2008; 8: 3. Available from: https://www.ncbi.nlm.nih. gov/pmc/articles/PMC2266699/.

12. Yong-Soon K, Jung-Eun M, Kyung-Taek L, In-Ho L, Tae-Jin K, $\mathrm{Ki}$-Heon $\mathrm{L}$, et al. Ovarian cancer during pregnancy clinical and pregnancy outcome. Korean Med Sci. 2010; 25 (2): 230-4.

13. Герасимова А. А., Гус А. И., Клименко П. А., авторы; Клименко Петр Афанасьевич, патентообладатель. Способ дифференциальной диагностики опухолевидных образований и опухолей яичников у беременных. Патент РФ № 2325118. 05.06.2007.

14. Герасимова А. А., Швырев С. Л., Соломатина А. А., Гус А. И., Клименко П. А. Способ выявления характера яичниковых образований. Онкология. 2013; 1: 34-40.

15. Tinelli R, Tinelli A, Tinelli F, Cicenelli E, Malvasi A. Conservative surgery for borderline ovarian tumors: a review. Gynecol Oncol. 2006; 100 (1): 185-91.

16. Манухин И. Б., Высоцкий М. М., Кушлинский Н. Е. Молекулярно-биологические факторы в патогенезе и

\section{References}

1. Battalova G. Ju. Pogranichnye opuholi jaichnikov (optimizacija metodov lechenija i mediko-socialnoj reabilitacii bolnyh) [dissertation]. M., 2005. Russian.

2. Novikova EG, Andreeva YuYu, Shevchuk AS. Fertility sparing treatment for patients with bilateral borderline ovarian tumors. Oncology. 2013; (1): 84-91.

3. Davydova IYu. Serous borderline ovarian tumors (clinical and morphological features, treatment, prognosis) [dissertation] M., 2018. Russian.

4. Giuntoli RL, Vang RS, Bristow RE. Evaluation and management of adnexal masses during pregnancy. Clin Obstet Gynecol. 2006; 49 (3): 492-505

5. Bakhidze EV. Ovarian tumors in pregnancy. Journal of Obstetrics and Women's Diseases. 2011; 3: 190-6.

6. Morice P, Uzan C, Gouy S, Verschraegen C, Haie-Meder C. Gynecological cancers in pregnancy. Lancet. 2012; 379 (9815); 558-69.

7. Aggarwal $\mathrm{P}$, Kehoe S. Ovarian tumors in pregnancy a literature review. Eur J Obstet Gynecol Reprod Biol. 2011; 155 (2): 119-24.

8. Gui T, Cao D, Shen K, Yang J, Fu C, Lang J, Liu X. Management and outcome of ovarian malignancy complicating pregnancy: an analysis of 41 cases and review of the literature. Clin Transl Oncol. 2013; 15 (7): 548-54.

9. Shloma EN, Fridman MV, Shelkovich SE, Demidchik YuE. Borderline epithelial tumors of the ovaries: clinical course and problems of morphological diagnosis. Minsk: Publishing house "Bel MAPO", 2012; 80 s. хирургическом лечении опухолей яичников. М.: Изд-во «Династия», 2007; 208 с.

17. Moghaddam SM, Amini A, Morris D, Pourgholami H. Significance of vascular endothelial growth factor in growth and peritoneal dissemination of ovarian cancer. Cancer Metastasis Rev. 2012; 31 (1-2): 143-62. DOI: 10.1007/s10555-011-9337-5.

18. Viallard C, Larrivée B. Tumor angiogenesis and vascular normalization: alternative therapeutic targets. Angiogenesis. 2017; 20 (4): 409-26. DOI: 10.1007/s10456-017-9562-9.

19. Новикова Е. Г., Шевчук А. С. Органосохраняющее лечение больных с пограничными опухолями яичников. Вопросы онкологии. 2014; 60 (3): 267-73.

20. Fauvet R, Brzakowski M, Morice P, Resch B, Marret H, Graesslin O, et al. Borderline ovarian tumors diagnosed during pregnancy exhibit a high incidence of aggressive features: results of a French multicenter study. Ann Oncol. 2012; 23 (6): 1481-7.

21. Zagouri F, Dimitrakakis C, Marinopoulos S, Tsigginou A, Dimopoulos MA. Cancer in pregnancy: disentangling treatment modalities. ESMO. 2016; 1 (3). Available from: https://www.ncbi. nlm.nih.gov/pmc/articles/PMC5070264/

22. Шевчук А. С. Повторные лапароскопические операции у больных со злокачественными опухолями яичников [диссертация]. М., 2005.

23. Kim JH, Kim TJ, Park YG. Clinical analysis of intra - operative frozen section proven borderline tumors of the ovary. J Gynecol Oncol. 2009; 20 (3): 176-80.

24. Brun JL, Cortez A, Rouzier R. Factors influencing the use and accuracy of frozen section diagnosis of epithelial ovarian tumors. Am J Obstet Gynecol. 2008; 199 (3): 241-7.

25. Du Bois A, Ewald-Riegler N, du Bois O, Harter P. Borderline tumors of the ovary - a systematic review. Geburtsh Frauenheilk. 2009; 69: 807-33.

26. Trope C, Davidson B, Paulsen T, Abeler VM, Kaern J. Diagnosis and treatment of borderline ovarian neoplasms «the state of the art». Eur J Gynecol Oncol. 2009; 30 (5): 471-82.

27. Fauvet $\mathrm{R}$, Poncelet $\mathrm{C}$, Boccara J. Fertility after conservative treatment for borderline ovarian tumors a French multicenter study. Fertil Steril. 2005; 83: 284.

28. Tinelli F, Tinelli R, La Grotta F. Pregnancy outcome and recurrence after conservative laparoscopic surgery for borderline ovarian tumors. Acta Obstet Gynecol Scand. 2007; 86: 81.

10. Davydova IYu, Kuznetsov W, Karseladze Al, Meshcheryakova LA. Borderline ovarian tumors. Obstetrics and gynecology: news, opinions, training. 2019; 7 (1): 92-104.

11. Nadereh B, Mojgan KZ, Mitra MG, Fatemeh G, Azamsadat M, Fahimeh G. Ovarian carcinoma with pregnancy: a clinicopathologic analysis of 23 cases and review of the literature. BMC Pregnancy Childbirth. 2008; 8: 3. Available from: https://www.ncbi.nlm.nih. gov/pmc/articles/PMC2266699/.

12. Yong-Soon $\mathrm{K}$, Jung-Eun $\mathrm{M}$, Kyung-Taek L, In-Ho L, Tae-Jin $\mathrm{K}$, $\mathrm{Ki}$-Heon $\mathrm{L}$, et al. Ovarian cancer during pregnancy clinical and pregnancy outcome. Korean Med Sci. 2010; 25 (2): 230-4.

13. Gerasimova AA, Gus Al, Klimenko PA, inventor; Klimenko Petr Afanasevich, assignee. A method for the differential diagnosis of tumorous formations and tumors of the ovaries in pregnant women. Russian Federation patent RF 2325118. 2007 Jun 1. Russan.

14. Gerasimova AA, Shvyrev S, Solomatina AA, Gus AI, Klimenko PA. Procedure for detecting the pattern of ovarian masses. Oncology. 2013; $1: 34-40$

15. Tinelli R, Tinelli A, Tinelli F, Cicenelli E, Malvasi A. Conservative surgery for borderline ovarian tumors: a review. Gynecol Oncol. 2006; 100 (1): 185-91.

16. Manuhin IB, Vysockij MM, Kushlinskij NE. Molekuljarnobiologicheskie faktory $v$ patogeneze i hirurgicheskom lechenii opuholej jaichnikov. M.: Izd-vo «Dinastija», 2007; 208 s. Russian.

17. Moghaddam SM, Amini A, Morris D, Pourgholami H. Significance of vascular endothelial growth factor in growth and peritoneal 
dissemination of ovarian cancer. Cancer Metastasis Rev. 2012; 31 (1-2): 143-62. DOI: 10.1007/s10555-011-9337-5.

18. Viallard C, Larrivée B. Tumor angiogenesis and vascular normalization: alternative therapeutic targets. Angiogenesis. 2017; 20 (4): 409-26. DOI: 10.1007/s10456-017-9562-9.

19. Novikova EG, Shevchuk AS. Organ-preserving treatment of patients with borderline ovarian tumors. Oncology issues. 2014; 60 (3): 267-73

20. Fauvet R, Brzakowski M, Morice P, Resch B, Marret H, Graesslin O, et al. Borderline ovarian tumors diagnosed during pregnancy exhibit a high incidence of aggressive features: results of a French multicenter study. Ann Oncol. 2012; 23 (6): 1481-7.

21. Zagouri F, Dimitrakakis C, Marinopoulos S, Tsigginou A, Dimopoulos MA. Cancer in pregnancy: disentangling treatment modalities. ESMO. 2016; 1 (3). Available from: https://www.ncbi. nlm.nih.gov/pmc/articles/PMC5070264/

22. Shevchuk AS. Repeated laparoscopic operations in patients with malignant ovarian tumors [dissertation]. M., 2005.
23. Kim JH, Kim TJ, Park YG. Clinical analysis of intra - operative frozen section proven borderline tumors of the ovary. J Gynecol Oncol. 2009; 20 (3): 176-80.

24. Brun JL, Cortez A, Rouzier R. Factors influencing the use and accuracy of frozen section diagnosis of epithelial ovarian tumors. Am J Obstet Gynecol. 2008; 199 (3): 241-7.

25. Du Bois A, Ewald-Riegler N, du Bois O, Harter P. Borderline tumors of the ovary - a systematic review. Geburtsh Frauenheilk. 2009; 69: 807-33.

26. Trope C, Davidson B, Paulsen T, Abeler VM, Kaern J. Diagnosis and treatment of borderline ovarian neoplasms «the state of the art». Eur J Gynecol Oncol. 2009; 30 (5): 471-82.

27. Fauvet R, Poncelet C, Boccara J. Fertility after conservative treatment for borderline ovarian tumors a French multicenter study. Fertil Steril. 2005; 83: 284.

28. Tinelli F, Tinelli R, La Grotta F. Pregnancy outcome and recurrence after conservative laparoscopic surgery for borderline ovarian tumors. Acta Obstet Gynecol Scand. 2007; 86: 81. 